\title{
Research on Reliability Selection and Application Control of Electronic Components
}

\author{
Jiahong Zhong, Yin Lei \\ Gannan Medical University, Ganzhou, 341000, China
}

Keywords: Reliability selection, Application control, Electronic components

\begin{abstract}
Electronic component is an important part of electronic system or electronic circuit. Once it fails, it may lead to the system or circuit failure, and then affect the reliability of electronic equipment. This article analyses the problems of reliability selection and application control of electronic components and gives solutions from the perspectives of optimizing purchase level, standardizing components utilization and enhancing storage of the capacity to provide some references for the relative researchers.
\end{abstract}

\section{Introduction}

Electronic component is a part of the whole machine to ensure the reliability of the performance of the machine from the electronic components. The reliability of electronic components mainly refers to the reliability and reliability of the application. The inherent reliability is mainly determined by the control of the design and manufacturing process and the quality of raw materials. The application of reliability refers to the role of electronic components in the whole system of electronic products, as far as possible to reduce the impact of human factors on the reliability of electronic products. How to choose how to use electronic components and electronic components are important indicators of the reliability of components, the production units are not the same, so the production line employees is different, even if they are in accordance with the same quality standards for production, its reliability will be different. When different units in the manufacture of electronic products, the selection of electronic components manufacturers are not the same, which led to the reliability of the product differences. Only the reliability of electronic components can meet the requirements of the relevant standards, in order to ensure the reliability of electronic products. Manufacturing process, manufacturing technology and materials will directly affect the inherent reliability of electronic components. The selection and application of electronic components are affecting the performance of the components and the reliability of the machine. It is not only related to the national defense related cutting-edge scientific research and the reliability of military electronic devices, but also related to people's production and life of the reliability of electronic products. In this case, it is very important to improve the system performance of the electronic equipment from the selection and application of components.

\section{Problems of Reliability Selection and Application Control of Electronic Components}

Impropriety of Purchase. Procurement progress cannot match the requirements of the product, resulting in a considerable loss of production cycle. When the electronic component quality problems, cannot get in touch with suppliers, resulting in two aspects of quality and progress cannot fully meet the requirements of the product. Component procurement should be carried out to ensure the quality, control progress, save money, as far as possible the focus of the policy, the change of the sale of components for active participation in the quality management of components. To clarify the composition of procurement documents, procurement list review requirements and procurement standards or norms of the main content. The inventory management is directly related to the quality of components. Therefore, it is necessary to clarify the storage conditions of components, warehouse management requirements, the establishment of components distribution system, the failure of the components of the management requirements. Now the vast majority of devices using laser marking 
or printing printing machine, using special chip clear handwriting, is not conspicuous, but not vague and difficult to erase, and re marking device to remove the original package mark, polished surface after a magnifying glass to observe carefully will have uniform grinding marks; and because must polished off the original tag, may burnish is deep, the overall thickness of such devices will be significantly less than the normal size, but not contrast or caliper measurement, generally difficult to distinguish. There are obvious signs of double. Removed from the abandoned components of the used components. It is generally more bright than genuine.

Impropriety of Utilization. Statistics show that: nearly half of the components failure is not the same as the reliability of the component itself is not high, but because of the improper choice of components. In addition, the improper application of components, but also easy to cause the failure of its electrical performance. Such as the use of the performance is not enough, did not take into account the amount of design, the test method is not appropriate, testing instruments, such as improper grounding, will greatly increase the failure rate of components. Faults of electronic components is largely to human factors directly or indirectly, because the electronic components are controlled by people from design, production to the whole process is put into use, so that the human factor is the analysis of failures of electronic components need to be considered. In the process of production, the storage, stacking and installation of the electronic components can be damaged due to the negligence of the staff. In simple terms, on the assembly process of electronic components, components in unit board after the adjustment of the whole system, if this time the machine runs smoothly, and electronic components and printed circuit board welding machine cannot run smoothly after. Through the inspection and analysis of the situation of the experts, the main reason is the electronic components and printed circuit board in the electric iron is no guarantee of good grounding condition for welding, the welding circuit in this case standard does not meet the requirements, will inevitably lead to the device is not working properly. When a radar product is put into use, if there is a crystal oscillator floating beyond the normal range of the situation, supervision and management personnel usually may think that this is because the electronic integrated circuit lead, but the replacement of integrated circuit cannot solve this problem, and again after the supervision of just understand the original circuit design of electronic components unreasonable. After the improvement of the circuit design, the unstable oscillation problem is solved.

Impropriety of Storage. Different types of components and storage cabinets. Label affixed to the door, the production directory for easy access. However, due to the good or bad of a lot of components cannot be seen from the appearance, such as capacitive leakage, chip breakdown, etc., after each experiment cannot be intuitive judgment and rejection. Just count on the number of re storage, resulting in the middle of the device is good or bad. This not only increases the workload of the late, also affect the practice effect compared to the experimental equipment, staff awareness of the protection of electronic components to many weak, often due to cause damage to components and the manager be negligent, it is difficult to site supervision, there is no strict restrictions in the issuance of backup devices, components loss rate is very high. If part of the components in the library management, is a complicated complex, especially the appearance of similar components easily confused, resulting in low efficiency of management. The purpose of setting up the standard outline of components is to effectively control the selection and use of components to achieve the reliability requirements of the components. Component standardization outline to the choice of components, use and control to make detailed provisions for all kinds of personnel rules. The establishment and implementation of the standardized outline of components directly reflects the level of reliability of a unit. Requirements of the components of the outline of the standardization of accessibility, operability and inspection. Accessibility is established according to the component standardization program after the implementation of the selection of each component to meet the requirements of reliability of the whole system, so that each component in a controlled state in the whole life cycle. 


\section{Countermeasures of Reliability Selection and Application Control of Electronic Components}

Optimize Purchase Level. The reliability test is usually performed only on the samples, which is designed for the characteristics of the components and the working conditions as well as the environmental conditions. In order to test and evaluate the reliability of the device, the internal defects of the components are fully exposed. The reliability test can be divided into two kinds: the special test and the evaluation of the service life of the component. Reliability test is very targeted, and requires a longer time and higher costs, so the choice of test items, should be targeted. Such as surface mount components in the machine, because when welding by thermal shock thermal stress, so it should be chosen as the thermal shock test, and scanning acoustic microscope examination in the test, to analyze its internal stratification, inspection and purchasing thermal stress; for the use of integrated package or reliability requirements the higher the equipment or system components, it is necessary to do accelerated life test, to test the reliability and stability of the high performance in the whole life cycle of equipment or system in. Reliability tests often bring internal damage to components, and these injuries can usually be accumulated, so the reliability of the test components are generally no longer used. Reliability test can also be used to optimize the quality of components and identify the authenticity of technology. In order to ensure the quality of the components on the computer, but also to save costs, in the procurement of components at all stages of the use of quality control technology should be focused on. In the selection of suppliers, the main implementation of reliability testing and physical analysis and inspection, which is to measure the stability of components and life, process conditions and stability is very important.

Standardize Components Utilization. Full load components, its life will be greatly reduced. In order to reduce the failure rate of components in the field, the use of components should be implemented to reduce the amount and cooling methods. At the same time, we should adopt margin design, redundant design to reduce the failure rate of components, improve the reliability of electronic components, thereby improving the reliability of the machine. Under the premise of ensuring the function of the circuit, we should try to replace the discrete devices with integrated circuits. For the special circuit, or by the high cost components of the circuit, can be added as a preventive measure of protection circuit. For the components with large power consumption, the radiator parts should be attached, and the contact is good. The correct choice of the installation process, such as the printed board with the mechanical hole, components of the installation hole, the size of the structure of rationality. In order to prevent the internal stress of components due to the unreasonable structure and process design, and accelerate the early failure of components. Want it timely to load and temperature when using the electronic components of the adjustment, at the same time in accordance with the redundancy design, margin design such measures to enhance the performance of components, reduce the failure rate; for more power components to say it is better to have the function of components corresponding to the radiator. Ensure that the circuit load does not exceed the standard of electronic components, of course, to ensure the good performance of the integrated circuit or electronic components have higher cost would be better. To speed up the reliability of the application of components, the preparation of the relevant components of the use of guidelines or rules, in order to improve the reliability of circuit design components of the design level. We can write files according to the actual situation of the unit, so that the design and use of personnel rules, will greatly reduce the human factors caused by component failure.

Enhance Storage Capacity. The component selection information database should be established and managed by the administrator, and the new information will be added in time. Selection and procurement of components. At the same time, in order to reduce the errors caused by human factors, it is necessary to provide software assurance, that is, the need for the design of specialized software to provide a design platform to ensure that the designer selects the device from the preferred library. Verify the information on the main performance parameters, the quality and reliability of the components, production control standards, test data reports, failure analysis report to be confirmed and verified. For the life cycle has been very short or discontinued product screening and replacement. With the development of China's new standards and the development of new products, a number of 
high quality and reliability standards and new products, has been able to meet the needs of equipment development, and have the ability to replace imported products. The implementation of the management mode, the staff can actively participate in the management and protection of components, the component loss rate is greatly reduced, and the causes of loss to be tracked for easy implementation of components of the training room management, compensation system, the training room components library can keep updated in real time. So the standard can be used to replace ordinary products, advanced new products to replace the old. The scoring system can be used to evaluate the performance level, the quality certification, the production execution standard, the quality level, the test condition, the reliability level and the usage history record. According to the principle and procedure of the evaluation, examination, examination and optimization of components, the principle of selection, retention and deletion of components is formulated.

\section{Conclusion}

With the rapid development of the electronics industry, more and more electronic equipment failures occur. Research shows that the vast majority of electronic products failures lie in the problem of components. It puts forward new requirements for the reliability of electronic components. We must strengthen the ability of purchase, utilization and storage to ensure the normal operation of electronic equipment.

\section{References}

[1] Gong Chaohui, Wang Bo, Circuit Design of the Use of the Reliability of Electronic Components [J]. Equipment Manufacturing Technology, 2011(3): 176-177.

[2] Wang Peng, Zhang Jinbiao, Reliability selection and application control of electronic components [J]. Electronic Test, 2016(9): 118-119.

[3] Zhong Weijun, LI Desheng, Cui Liang, A Method for the Reliability Prediction of Electronic Components in Guided Munitions [J]. Equipment Environmental Engineering, 2014, 11(2): 103-105.

[4] Yu Haiyan, Standardized Application Management of Electronic Components [J]. Electronic Science \& Technology, 2015, 2(3): 372-378. 